\title{
Barriers and enablers of COPD telerehabilitation - a frontline staff perspective
}

This article was published in the following Dove Press journal: International Journal of COPD

\section{Christina Sadolin Damhus' Christina Emme 2 Henrik Hansen' \\ 'Research Unit of Chronic Diseases and Telemedicine - Center for Clinical Research and Prevention, Bispebjerg and Frederiksberg University Hospital, Copenhagen, Denmark; ${ }^{2}$ Department of Quality and Education, Bispebjerg and Frederiksberg University Hospital, Copenhagen, Denmark}

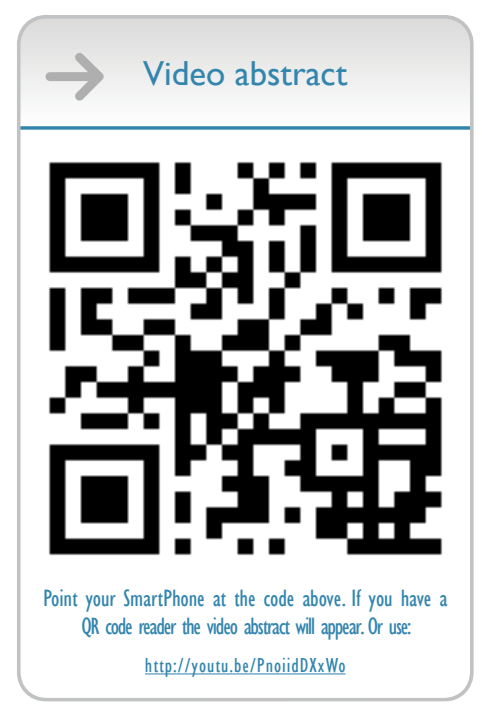

Correspondence: Henrik Hansen Research Unit of Chronic Diseases and Telemedicine - Center for Clinical Research and Prevention, Bispebjerg and Frederiksberg University Hospital, Bispebjerg Bakke 23, 2400 Copenhagen NV, Denmark

Tel +45 28946780

Email henrik.hansen.09.regionh.dk
Background: Telerehabilitation (TR) aimed at patients with COPD has shown promising effects on symptoms, physical function, and quality of life, but little research has been conducted to understand the impact of implementation on frontline health professionals. Therefore, the aim of this study was to examine the barriers and enablers of health professionals to online exercisebased TR in patients with COPD, to support a successful implementation process.

Methods: Semistructured individual and focus group interviews were conducted with 25 health professionals working with conventional COPD rehabilitation or TR. Interviews were audiotaped and transcribed verbatim. Investigator triangulation was applied during data generation. The Theoretical Domains Framework directed the interview guide and was used as a coding framework in the analysis.

Results: We identified six predominant domains essential in understanding the enablers and barriers of TR from a staff perspective: 1) skills, 2) professional role and identity, 3) beliefs about capabilities, 4) beliefs about consequences, 5) environmental context and resources, and 6) social influences. We found that health professionals held both enablers and barriers important for the implementation process of TR. TR introduces new work tasks and new ways for the health professionals to communicate and exercise with the patients, which influence their professional role and self-perceived capability.

Conclusion: Specific attention toward involvement of the health professionals in the decision process combined with sufficient education and skill training is highly essential to support a successful implementation of TR in clinical practice.

Keywords: telecare, health professionals, theoretical domains framework, qualitative research, implementation process

\section{Background}

COPD is a significant and growing public health concern and a leading cause of morbidity and mortality. ${ }^{1}$ COPD is the third leading cause of death worldwide and approximately three million people die of COPD every year., ${ }^{2,3}$ COPD is incurable, but both pharmacological and nonpharmacological treatment is essential to improve or maintain patients' quality of life and functional status. ${ }^{1}$ Optimal treatment focuses on medication management, patient education, action plans for exacerbations, and pulmonary rehabilitation (PR). ${ }^{1}$ Exercise training with respect to both exercise tolerance and symptoms of dyspnea is a cornerstone of PR and has been reported to have consistently high clinical efficacy. ${ }^{4-7}$ Currently, most of these exercise programs are hospital based and patients are expected to perform exercise sessions with regular supervision and monitoring to achieve persistent and optimal benefits. ${ }^{5-8}$ However, adherence and maintenance are major challenges to the success of these rehabilitation programs, and high dropout rates are 
an additional challenge in PR. ${ }^{9-12}$ Barriers such as lack of mental and physical resources, disruption of daily routines, distance to the training venues, and transportation difficulties are often reported by COPD patients as reasons for not attending the programs..$^{9,11,13}$ To overcome these barriers, different telecare interventions have been carried out in various ways demonstrating the potential in reducing emergency department visits, mortality rates, and health care costs. ${ }^{14-17}$ In this article, the term telecare is not limited to describe one type of intervention but covers a wide range of interventions, applying telephone calls, apps, or computer screens to monitor, educate, or train the patients. However, evidence indicating the effectiveness and cost-effectiveness of telecare remains mixed and difficult to synthesize. ${ }^{18,19}$ This is likely due to the contextual heterogeneity of studies evaluating telecare across factors, including the type of intervention, the participants involved, and the health care system in which they are located. ${ }^{18-20}$ Importantly, qualitative research has begun to investigate how telecare is experienced from the perspective of both COPD patients and health professionals. ${ }^{21-24}$ In general, most of these studies have focused on the satisfaction, adherence, and acceptability of telecare interventions from the perspective of the patients resulting in conclusions, which support an increased use of telecare. ${ }^{21,23}$ Studies taking the health professionals' perspective have on the other hand identified a series of barriers when implementing telecare interventions. Often identified barriers are lack of organizational support and resources, telecare experienced as threatening due to changes in work routines, workload and skills set, as well as equipment reliability. ${ }^{25-27}$ Furthermore, the health care professionals' overall attitude toward telecare was identified as essential to the adaption and acceptance of the interventions..$^{25,28}$ Therefore, overcoming these potential barriers is key to a successful implementation of telecare and an important step to support effective telecare interventions. To identify these potential barriers, the Theoretical Domains Framework (TDF) has previously been applied as it supports the identification of barriers and enablers in implementation of health interventions. ${ }^{13,29,30}$ A growing body of literature in the field of implementation theory suggests that frontline staff's attitudes and resources are of great importance when new health interventions are to be implemented. ${ }^{31,32}$ The importance of the frontline staff's perspective has been acknowledged in previous studies investigating the implementation of different telecare interventions. However, these studies were performed in the context of telecare that facilitates communication or monitoring but did not include online exercise training. ${ }^{20,23,26}$ In this article, telecare that includes a supervised, online exercise session to patients with COPD is defined as telerehabilitation (TR). To our knowledge, no previous studies have investigated how TR is experienced from the perspective of the health care professionals. Therefore, the aim of this study was to examine the barriers and enablers of health professionals to online exercise-based TR in patients with COPD, to support a successful implementation process.

\section{Methods}

This study had a qualitative design that enabled us to get insight to the participants' thoughts and attitudes toward TR. The study applied the TDF developed by Michie et al. ${ }^{29}$ The aim of TDF is to simplify and integrate behavior change theories into a single framework, making theory more accessible and applicable to other disciplines. The framework is developed by a research group of health psychologists and implementation researchers who synthesized 33 theories and 128 key theoretical constructs related to behavior change. ${ }^{29}$ Their work resulted in the first edition of the TDF consisting of 12 domains. In 2012, the TDF was validated to confirm the optimal domain structure, content, and labels. ${ }^{33}$ In this study, we applied the validated version of TDF that consists of 14 domains (Table 1).

Table I The theoretical domains framework

\begin{tabular}{ll}
\hline Theoretical domains & Examples of interview questions \\
\hline Knowledge & What do you know about TR? \\
Skills & Do you know how to perform TR? \\
Professional role and & Is TR a part of your professional role? \\
identity & \\
Beliefs about capabilities & To what extent do you feel capable of \\
& doing TR? \\
Optimism & Do you believe TR can make a \\
Beliefs about & difference to the patients? \\
consequences & What are the enablers of TR? \\
Reinforcement & What are the barriers of TR? \\
Intentions & Which factors can motivate you to \\
Goals & perform TR? \\
Is performing TR of importance to you? & What can you achieve when performing \\
Memory, attention, and & TR? \\
Environmental context & Are there elements difficult to \\
and resources & remember in TR? \\
Social influences & Do you have the necessary resources to \\
Emotions & perform TR? \\
\hline Which attitudes do your colleagues hold \\
about TR?
\end{tabular}

Note: Theoretical domains from Cane et al. ${ }^{33}$

Abbreviation: TR, telerehabilitation. 


\section{Ethics}

This study is in accordance with the ethical principles of the Helsinki Declaration. ${ }^{34}$ The Danish Data Protection Agency approved the research database (id: BFH-2017-021). In Denmark, approval from the Research Ethics Committee is only required for interventional studies. Because this study is a noninterventional study, an ethical approval was not necessary. Prior to the interviews, the aim of the study and the participants' role in the research project were fully explained verbally and in writing. The participants agreed to the interviews being audiotaped with their statements anonymized, and informed consent was obtained from all the participants.

\section{Participants}

In a Danish setting, COPD rehabilitation is performed by nurses and physiotherapists why these two health professions were selected for this study. We recruited two groups of health professionals. A group with no-tele experience and a teleexperienced group. This was done so we were able to relate their attitudes toward TR at a later point. In the no-tele-experienced group, an inclusion criterion was a previous experience providing COPD rehabilitation either patient education or exercise training. In the tele-experienced group, participants were only included if they had experience applying exercise or education to COPD patients via real-time video telemedical technology. Participants were recruited through a Danish COPD network and by contacting gatekeepers who provided access to the health professionals. Once gatekeepers had identified initial participants, a snowballing technique was used to expand the variety of the sample. ${ }^{35}$ The recruitment process ended when data saturation was obtained. ${ }^{36}$

\section{Data generation}

The data included four focus group interviews with health professionals having no TR experience and two focus group interviews and three individual interviews with participants having TR experience (Table 2). The semistructured interview guide was based on the 14 domains in TDF and was designed to explore barriers and enablers regarding TR. The interview guide was initially pilot tested in a focus group interview, which confirmed the relevance of the questions and resulted in minor modifications of the interview guide. Interviews took place in a location convenient and easily accessible to the participants. This included hospitals and rehabilitation centers in Denmark. All interviews were conducted by the first author (CSD), while the second (CE) and third authors $(\mathrm{HH})$ observed the interviews, wrote field notes, and asked supplementary questions during the interviews. The focus group interviews and the individual interviews lasted between 50 and 70 minutes with a mean duration of 1 hour. The interviews were audio recorded and further transcribed using the Express Scribe Transcription Software version 6.00 (NCH Software, Inc., Canberra, Australia).

\section{Data analysis}

Initially, the transcripts were analyzed using content analysis to identify meaning units in the data. ${ }^{37}$ This included an open coding of each interview, resulting in a number of meaning

Table 2 Participants and interview characteristics

\begin{tabular}{|c|c|c|c|c|c|c|}
\hline \multirow[t]{2}{*}{ No interview } & \multirow{2}{*}{$\begin{array}{l}\text { Type of } \\
\text { interview }\end{array}$} & \multirow{2}{*}{$\begin{array}{l}\text { TR experience } \\
\text { (Yes/no) }\end{array}$} & \multicolumn{3}{|c|}{ Education } & \multirow[t]{2}{*}{ No participant } \\
\hline & & & Nurses & Physi & & \\
\hline $\mathbf{I}$ & Focus group & No & $x x$ & $x$ & 3 & \multirow{4}{*}{14} \\
\hline 2 & Focus group & No & - & $x x x$ & 3 & \\
\hline 3 & Focus group & No & $x$ & $x x$ & 3 & \\
\hline 4 & Focus group & No & $x$ & $x x x x$ & 5 & \\
\hline 5 & Focus group & Yes & $x$ & $x x$ & 3 & \multirow{5}{*}{ I } \\
\hline 6 & Focus group & Yes & $x$ & $x x x x$ & 5 & \\
\hline 7 & Individual & Yes & - & $x$ & I & \\
\hline 8 & Individual & Yes & - & $x$ & I & \\
\hline 9 & Individual & Yes & - & $x$ & I & \\
\hline \multicolumn{3}{|c|}{ Participants in total } & 6 & 19 & 25 & \\
\hline \multicolumn{3}{|l|}{ Gender N (\%) } & & & \multirow[b]{2}{*}{$2(8)$} & \\
\hline \multicolumn{2}{|l|}{ Male } & & & & & \\
\hline \multicolumn{2}{|l|}{ Female } & & & & $23(92)$ & \\
\hline \multicolumn{3}{|c|}{ Age in years (range) mean } & \multicolumn{3}{|c|}{$(24-57) 42.2$} & \\
\hline \multicolumn{3}{|c|}{ Years of education (range) mean } & $(0.6-32$ & & & \\
\hline
\end{tabular}

Abbreviation: TR, telerehabilitation. 
units. These meaning units were then applied in a deductive analysis in which TDF was used as a coding framework. ${ }^{38}$ First, two of the authors (CSD and $\mathrm{HH}$ ) reviewed the meaning units and divided them into the 14 domains. Second, they discussed and agreed on the coding. In the few circumstances where disagreement occurred, a third author (CE) contributed to the discussion and had the final say. Meaning units were not restricted to one domain but were cross-indexed when they were relevant to more than one domain. The meaning units were clustered into categories which were ordered into subthemes in each domain. A summary of each domain was written, and the most predominant domains were identified for this article (Table 3).

\section{Results}

A total of 25 health professionals participated in this study; 6 nurses and 19 physiotherapists (24-57 years) all working with TR or conventional COPD rehabilitation in either hospitals or municipalities in Denmark. Six focus groups and three single interviews were conducted and analyzed, resulting in six predominant domains; 1) skills, 2) professional role and identity, 3) beliefs about capabilities, 4) beliefs about consequences, 5) environmental context and resources, and 6) social influences (Table 3 ).

\section{Skills}

\section{Communication skills}

Some participants in the no-tele-experienced group perceived the screen as a barrier to communicate with patients. They presumed that the patients would interrupt each other when speaking on the screen or that guiding the patients would be time-consuming compared with current practice. Furthermore, some participants mentioned that they would need to learn how

Table 3 Domains and subthemes

\begin{tabular}{ll}
\hline Domains & Subthemes \\
\hline Skills & $\circ$ Communication skills \\
& $\circ$ A need for creative health professionals \\
Professional role and & $\circ$ Getting the right type of rehabilitation to \\
identity & the right patients \\
& $\circ$ Online vs physical meeting with the patients \\
Beliefs about capabilities & $\circ$ Feeling safe when performing TR \\
Beliefs about & $\circ$ Interpersonal communication and relations \\
consequences & on screen \\
& $\circ$ Performing rehabilitation with no exercise \\
Environmental context & $\circ$ Transportation \\
and resources & $\circ$ Resources \\
Social influences & $\circ$ Cooperation with other health \\
\end{tabular}

Abbreviation: TR, telerehabilitation. to express themselves loud and clear on a screen. Likewise, participants in the group with tele experience emphasized that they had developed new communication skills to perform rehabilitation on a screen. A participant said:

When I started doing TR I had to get used to the fact that Mrs Jensen was sitting in the other end of the region but all our communication was on the screen. So I realized that I had to change my choice of words so I could explain the exercises to her in a more visual way. I think that was the greatest difference compared to conventional rehabilitation. [...]

In addition, the tele-experienced group emphasized that communication on the screen had been a challenge in the beginning but had become a natural way to communicate with the patients. A tele-experienced participant mentioned to this topic that she had been glad to receive education in how to communicate on a screen before performing TR.

\section{A need for creative health professionals}

The majority of the participants within the no-tele-experienced group presumed that performing TR would call for a more creative approach to exercise training than their current practice, eg, to develop a rehabilitation program that could be applied online. A no-tele-experienced participant said:

[...] I could use some inspiration in which exercises to use online, I would like to see someone else do it before we begin, that would give me some inspiration.

\section{Professional role and identity}

\section{Getting the right type of rehabilitation to the right} patients

All participants agreed that TR is a task that belongs within their field of work as nurses or physiotherapists. However, the health professionals emphasized that TR should not replace conventional COPD rehabilitation and that allocating the right type of rehabilitation to the patients was an essential part of their professional role. A tele-experienced participant said:

Perhaps I already said this, but I think what matters the most to me is to offer telerehabilitation to the right group of patients. We should not just apply it to all patients, because we are able to. We have to make sure it is used in a meaningful way.

In addition, the tele-experienced participant explained that to have the final say about which patient group to offer TR, had increased work satisfaction. The participant said:

First, I was asked if I wanted to implement TR to young, in-work COPD patients. But I suggested that the most fragile COPD patients would benefit the most. So I got the 
opportunity to design and develop it and I am really proud to work with TR.

According to the participants, the target group of TR is the COPD patients with the worst health status and transportation difficulties, which might hinder their participation in conventional rehabilitation. The tele-experienced group emphasized that TR made it possible for them to reach these groups. A participant said:

Telerehabilitation provides an opportunity to exercise with a group of patients I otherwise would not see, because they are too fragile to participate in conventional rehabilitation. It is this specific group we would lose if we couldn't offer them this (telerehabilitation). That is for sure a huge benefit.

\section{Online vs physical meeting with the patients}

The no-tele-experienced group emphasized that they would prefer to perform physical, conventional COPD rehabilitation instead of TR. A common explanation was the barrier to get an interpersonal connection with the patients on the screen. A participant said:

The personal connection would never be the same on a screen. Even though I am able to talk with the patient on the screen, it would never be the same as sitting in front of someone. [...]

In contrast, participants from the tele-experienced group could not relate to this issue, but most of them acknowledged the benefit of having an individual meeting with the patients before TR. Another barrier of the screen was the concern of being able to read the patients' symptoms online. A participant said:

If I see a patient with dyspnea, holdings on his knees after few meters of walking, I would immediately think that he needs a walker because then he can increase his walking distance. But I think it is these small observations we will miss if we do not see the patients in real life. I feel that I can help the patients more if I have looked them in the face.

In contrast, a tele-experienced participant said the following about the physical meeting:

I do not think it was a must. It was not the physical meeting that was essential to how I connected with the patients [...] and $[\ldots]$ to me it would be fine to have all the conversations on the screen.

\section{Tasks not included in professional role}

The participants did not think that fixing technical issues or installing tele equipment should be a part of their job or professional role. Furthermore, technical issues were the most often mentioned barrier. The participants in the no-tele-experienced group presumed that they would not get sufficient IT support and were concerned that they would have to fix technical issues themselves. A participant said:

As long as it is not our job to install the screen in the patients' home and fix technical issues, telerehabilitation is a nice alternative.

All the participants in the tele group had experienced technical issues and described them as frustrating and as barriers to perform TR. Moreover, a no-tele-experienced nurse emphasized that TR would be a "must do" task because the nurse would like to focus on the patients and nursing and no other tasks, such as fixing technical issues and introducing patients in using a screen.

\section{Beliefs about capabilities}

Feeling safe when performing TR

In general, all participants emphasized that they felt capable of doing TR and some with tele experience indicated that after some practice they found themselves good, competent, and safe when performing TR. However, comments from the notele-experienced indicated that they did not feel completely safe about TR. One participant said:

The greatest barrier is that I am not physically there to help the patient. Not only when we exercise but also in case the patient's condition gets worse.

This barrier resulted in more participants saying that they would push the patients less when exercising. A participant said:

You will push them less when you are not in the same room as the patients. You are not able to help them the only way is to call an ambulance.

In contrast, the majority of the tele-experienced group was not afraid to physically challenge the patients. A participant said:

I feel that I push them just as much as the patients I see in the rehabilitation center, but still, the patients know they have the final say and if they feel the need to sit down and take a break even though I say they can do more exercises, that's the way it is.

\section{Beliefs about consequences Interpersonal communication and relations on screen}

A general presumption in the no-tele-experienced group was the screen as a barrier for the patients to interact, eg, to give 
each other advice and cheer each other up. Furthermore, they believed that some social situations would not be possible on the screen. A no-tele-experienced participant said:

In telerehabilitation we will miss the kind of situation where Caren brings a bar of chocolate and shares it out and we all laugh about John who has difficulties in opening the package. It gives something to the group.

However, these kinds of "missing situations" were not mentioned in the tele-experienced group. Instead, they emphasized how social interactions happened between the patients on the screen. Two participants said independently:

$[\ldots]$ there was a really nice dynamism in the group. No one was being bullied. They cheered each other on and encouraged each other to exercise, so it was nice to see that they cared so much for each other.

And:

I have experienced that even though the patients had rehabilitation on the screen they began to see each other outside the rehabilitation program - that is fantastic.

\section{Performing rehabilitation with no exercise equipment}

A barrier of TR, often mentioned by the no-tele-experienced participants, was to provide sufficient rehabilitation when patients were located in their home with no access to training venues or equipment. None of the participants in the teleexperienced group could relate to this issue as they believed they provided an efficient exercise session with simple equipment. A tele-experienced participant said:

The patients do not need training equipment to exercise in their home. They can use water bottles or stones. They do not have to buy training equipment.

\section{Environmental context and resources Transportation}

All participants agreed that a great enabler of TR is that the patients do not need the long transportation and waiting time, which is undesirable and exhausting for the COPD patients. A participant said:

It is the transportation time that is a great enabler. Today, I just had a patient saying; "damn, it is so great that I do not have to come in here". Some people like it and others do not. I know some patients who do not want telerehabilitation. They would rather come to the rehabilitation center even though they have to drive. They like to come and talk to people because they sit at home, doing nothing anyway.

\section{Resources}

In the no-tele-experienced group, there was a common understanding that adequate resources would not be available if TR was implemented in their department. The no-teleexperienced participants presumed that hiring IT staff to install and introduce the patients to the screen would not be prioritized. This was believed to produce an unequal access to TR. Two no-tele-experienced participants said:

Then it is only the intelligent patients and the patients that can afford a screen who can get TR and not the patients who actually need it.

And:

Yes, it would only be the patients with grandchildren able to help them and smart patients who are able to do it themselves.

\section{Social influences}

\section{Cooperation with other health professionals about TR}

The participants emphasized that a close collaboration with colleagues and other health professionals, such as doctors and managers, is essential to perform TR. Most participants said that their board of directors in general was favorable to TR but did not prioritize it as much as conventional COPD rehabilitation. Other participants mentioned how they had experienced issues when cooperating and communicating with doctors about TR. A participant said:

In our group we struggle with the medical doctors. They believe there is no evidence to prove the benefit of telemedicine, so we have no agreement about this across health professions. TR is driven solely by nurses and physiotherapists. The doctors do not want to be a part of it. They just ask why we have to spend all this money on it. I think it is a challenge that no medical doctors want to take part in this.

\section{Discussion}

This qualitative study found that health professionals in general had a positive attitude toward TR, but at the same time relevant enablers and concerns were identified during the analysis. The barriers and enablers were in particular prominent within the following domains: 1) skills, 2) professional role and identity, 3) beliefs about capabilities, 4) beliefs about consequences, 5) environmental context and resources, and 6) social influence.

In spite of the growing use of telecare interventions to manage the monitoring, treatment, and rehabilitation of COPD patients, studies focusing solely on the frontline staff 
perspective on telecare are limited..$^{28,39}$ The majority of relevant studies have investigated the attitude to different telecare interventions from both patient and staff perspective. ${ }^{40-43}$ These studies found that health professionals in general experienced more barriers related to telecare than patients. Such results might be biased due to the characteristics of included patients within the studies. Only patients who are motivated or presume that TR would be beneficial choose to participate in this type of study. Furthermore, a mixed-method systematic review found that studies with this double perspective focused on patient view to the detriment of detailed analysis of staff perspective..$^{28}$ Our study is therefore important as it has a staff perspective only.

Our findings add to the current literature as we identified barriers and enablers that previously have been reported in the context of telecare. First, several studies found that health professionals reported technical tasks as a great barrier to their job and professional role. ${ }^{20,26,44-47}$ Similarly, the participants in this study experienced that technical tasks increased their preexisting work burden and undermined their professional identity. Second, more studies emphasized that telecare would not be able to replace the physical meeting because important communication would be missed. ${ }^{25,27}$ In addition, a study by Sølling et al found that it was emphasized by the patients and the health professionals that a personal relationship must be established before meeting online..$^{45}$ This was also a central barrier in our study as the no-tele-experienced group doubted whether they could communicate and read the patients' symptoms online and requested the physical meeting before meeting online. However, some of our tele-experienced participants did not agree on this, as they emphasized how after some time they had been able to communicate and create a good connection with the patients online. Moreover, in our study, it was essential to the participants that TR was implemented as a supplement to conventional COPD rehabilitation as the participants believed that TR would be the right type of rehabilitation to many COPD patients but not to all. Also, the participants' professional role and judgment were decisive when patients were allocated to different rehabilitation programs. Similarly, a study by Rykkje et al found that health professionals emphasized their responsibility to meet the patient's individual needs. ${ }^{27}$ Furthermore, they concluded that future telecare should not be restricted to specific patient groups but target the most eligible candidates.

One central enabler emphasized by both the no-teleexperienced and the tele-experienced participants in our study was the potential of TR to target a group of patients that otherwise would not participate due to poor health or long transportation to the rehabilitation center. Similarly, a study by Inskip et al found that health professionals reported that being able to support patients who otherwise would not participate in rehabilitation was satisfying and made their job meaningful. ${ }^{47}$ Likewise, we found that making a difference to the patients was an often mentioned motivator to perform TR.

As our study adds to the emerging literature on telecare, it also provides new knowledge. Our study design enabled us to relate attitudes from health care professionals with and without COPD tele experience. We found that the teleexperienced group had fewer barriers and more enablers regarding TR than the no-tele-experienced group. In addition, the no-tele-experienced group reported barriers in training patients online because of the absence of training facilities in the patient's home. This was not a barrier to the experienced group. As previous studies are performed in the context of telecare that facilitate communication or monitoring but do not include exercise training, we were not able to directly compare these exercise-specific barriers to the findings of previous work.

\section{Strengths and limitations}

The strengths of the study relate to the use of a validated theoretical framework. TDF was beneficial for the purpose of this study as it is developed especially to access enablers and barriers for implementation.

Several steps have been made to ensure trustworthiness of our study ${ }^{48}$ In the analysis, the authors both independently and interdependently coded, analyzed, and discussed the results (investigator triangulation) ${ }^{49}$ Prior to this study, we wrote down our presumptions and hypothesis. This increased the conformability of the study as it allowed us to be reflective about our own role in the data construction. Furthermore, we used both focus groups and single interviews. This combination ensured both an in-depth and a broad data material as the single interviews enabled us to explore specific opinions and experiences expressed in the focus groups ${ }^{50}$ Furthermore, the participants in the focus groups both shared experiences and explained themselves to each other, which made the focus group more than the sum of separate individual interviews. ${ }^{51}$ We conducted six focus groups and three single interviews including 25 participants. The participants differed in education, tele-experience, and place of employment, which supports a high credibility. Our sample represents the majority of health professionals having COPD TR experience in Denmark. This is a strength to this study as participants have been recruited from four of the five regions of Denmark. A potential limitation of this study is the size of the focus 
groups (three to five participants) as 6 to 12 persons are often recommended in the literature. ${ }^{50,52}$ However, only few health professionals in Denmark have experience with TR, which was the main reason for the relatively small focus groups. Small groups may limit the range of viewpoints and impede the synergistic effect. However, participant interaction is the hallmark of the focus group method, and our groups were dynamic with all participants contributing to the conversation that make the method suitable for this study. ${ }^{53}$

No new information emerged after the eighth interview, which indicates data saturation. To establish the dependability of our study, we ensured consistency during the data collection and analysis by conducting all the interviews in a similar manner and by applying a systematic code-recode system.

The transferability of the findings is challenged by the context of the study and the design of the teleintervention. In Denmark, there is no standardized definition of what TR must include. Therefore, our participants referred to similar but not identical interventions when they discussed TR. Still, all the tele-experienced participants in this study referred to tele interventions that included online exercise sessions and in some cases patient education.

\section{Implications for future research, policy, and practice}

To our knowledge, this is the first study to examine the barriers and enablers of frontline health professionals to online TR in patients with COPD. The study is therefore an important contribution to the literature as it identifies barriers and enablers important to the implementation process of COPD TR.

The success of telecare depends significantly on the frontline staff's attitude toward it. Therefore, further research is needed within this area. Based on our findings, future research should focus on how the frontline staff's professional role and identity is influenced or compromised by TR. We found that the majority of the health professionals prefer to physically meet the patients before rehabilitation online. This calls for further research to examine what is lost when we replace face-to-face communication with telecommunication. Furthermore, we found that technical tasks related to TR were emphasized as a great barrier and undermined the staff's professional role. Therefore, when implementing new teleinterventions, it is crucial that health professionals get a sufficient introduction to the tele devices and are assured that IT support is available when needed..$^{39,47}$

Moreover, we found that the tele-experienced group held a more positive attitude toward telecare than the no-experienced group. Most participants in the tele-experienced group had years of experience with TR and had been included in the development of the teleinterventions. This indicates that to include health professionals in the decision process might increase their satisfaction with TR. ${ }^{39}$ Furthermore, it indicates that it takes time to successfully implement TR as experience is needed before the frontline staff feels comfortable with their new tasks related to TR.

In addition, the findings of this study suggest that TR is not just another way of delivering existing health care. TR introduces new work tasks and is a different way of providing care that redefines the health professionals' identity. Therefore, education in how to communicate and exercise on a screen must not be neglected as these elements are essential to support a successful implementation of TR.

\section{Conclusion}

Health professionals hold both enablers and barriers important to the implementation process of TR. TR introduces new work tasks and new ways for the health professionals to communicate and exercise with the patients, which influences their professional role and self-perceived capability. Specific attention toward involvement of the health professionals in the decision process combined with sufficient education and skill training is highly essential to support a successful implementation of TR in clinical practice.

\section{Disclosure}

The authors report no conflicts of interest in this work.

\section{References}

1. Agusti A, Hurd S, Jones P, Fabbri LM. Global initiative for chronic obstructive lung disease. Available from: http://goldcopd.org/gold-2017global-strategy-diagnosis-management-prevention-copd/. Accessed January 8, 2018.

2. WHO. Chronic respiratory diseases. Chronic respiratory diseases. Available from: http://www.who.int/respiratory/copd/burden/en/. Accessed January 8, 2018.

3. Lozano R, Naghavi M, Foreman K, et al. Global and regional mortality from 235 causes of death for 20 age groups in 1990 and 2010: a systematic analysis for the Global Burden of Disease Study 2010. The Lancet. 2012; 380(9859):2095-2128.

4. Liu WT, Wang CH, Lin HC, et al. Efficacy of a cell phone-based exercise programme for COPD. Eur Respir J. 2008;32(3):651-659.

5. Spruit MA, Singh SJ, Garvey C, et al. An official American Thoracic Society/European Respiratory Society statement: key concepts and advances in pulmonary rehabilitation. Am J Respir Crit Care Med. 2013;188(8):e13-e64.

6. Puhan MA, Gimeno-Santos E, Cates CJ, Troosters T, Scharplatz M, Walters EH, Steurer J. Pulmonary rehabilitation following exacerbations of chronic obstructive pulmonary disease. Cochrane Database Syst Rev. 2016;12:CD005305.

7. Mccarthy B, Casey D, Devane D, Murphy K, Murphy E, Lacasse Y. Pulmonary rehabilitation for chronic obstructive pulmonary disease. Cochrane Database Syst Rev. 2015;2(2):CD003793. 
8. Rugbjerg M, Iepsen UW, Jørgensen KJ, Lange P. Effectiveness of pulmonary rehabilitation in COPD with mild symptoms: A systematic review with meta-analyses. Int J Chron Obstruct Pulmon Dis. 2015; 10:791-801.

9. Keating A, Lee A, Holland AE. What prevents people with chronic obstructive pulmonary disease from attending pulmonary rehabilitation? A systematic review. Chron Respir Dis. 2011;8(2):89-99.

10. Bjoernshave B, Korsgaard J, Jensen C, Vinther Nielsen C. Participation in pulmonary rehabilitation in routine clinical practice. Clin Respir J. 2011;5(4):235-244.

11. Bjoernshave B, Korsgaard J, Nielsen CV. Does pulmonary rehabilitation work in clinical practice? A review on selection and dropout in randomized controlled trials on pulmonary rehabilitation. Clin Epidemiol. 2010;2:73-83.

12. Fischer MJ, Scharloo M, Abbink JJ, et al. Drop-out and attendance in pulmonary rehabilitation: The role of clinical and psychosocial variables. Respir Med. 2009;103(10):1564-1571.

13. Cox NS, Oliveira CC, Lahham A, Holland AE. Pulmonary rehabilitation referral and participation are commonly influenced by environment, knowledge, and beliefs about consequences: a systematic review using the Theoretical Domains Framework. J Physiother. 2017;63(2): 84-93.

14. Chaplin E, Hewitt S, Apps L, et al. Interactive web-based pulmonary rehabilitation programme: a randomised controlled feasibility trial. $B M J$ Open. 2017;7(3):e013682.

15. Tsai LL, Mcnamara RJ, Moddel C, Alison JA, Mckenzie DK, Mckeough ZJ. Home-based telerehabilitation via real-time videoconferencing improves endurance exercise capacity in patients with COPD The randomized controlled TeleR Study. Respirology. 2017;22(4): 699-707.

16. Bourne S, Devos R, North M, et al. Online versus face-to-face pulmonary rehabilitation for patients with chronic obstructive pulmonary disease: randomised controlled trial. BMJ Open. 2017;7(7):e014580.

17. Vasilopoulou M, Papaioannou AI, Kaltsakas G, et al. Home-based maintenance tele-rehabilitation reduces the risk for acute exacerbations of COPD, hospitalisations and emergency department visits. Eur Respir J. 2017;49(5):1602129.

18. Cruz J, Brooks D, Marques A. Home telemonitoring in COPD: A systematic review of methodologies and patients' adherence. Int $J$ Med Inform. 2014;83(4):249-263.

19. Thokala P, Baalbaki H, Brennan A, et al. Telemonitoring after discharge from hospital with heart failure: cost-effectiveness modelling of alternative service designs. BMJ Open. 2013;3(9):e003250.

20. Hunting G, Shahid N, Sahakyan Y, et al. A multi-level qualitative analysis of Telehomecare in Ontario: challenges and opportunities. BMC Health Serv Res. 2015;15(1):544.

21. Hoaas H, Andreassen HK, Lien LA, Hjalmarsen A, Zanaboni P. Adherence and factors affecting satisfaction in long-term telerehabilitation for patients with chronic obstructive pulmonary disease: a mixed methods study. BMC Med Inform Decis Mak. 2016;16(1):26.

22. Vatnøy TK, Thygesen E, Dale B. Telemedicine to support coping resources in home-living patients diagnosed with chronic obstructive pulmonary disease: Patients' experiences. J Telemed Telecare. 2016; 01357633X(1): 15626854 .

23. Dinesen B, Andersen SK, Hejlesen O, Toft E. Interaction between COPD patients and healthcare professionals in a cross-sector tele-rehabilitation programme. Stud Health Technol Inform. 2011;169:28-32.

24. Seidman Z, Mcnamara R, Wootton S, et al. People attending pulmonary rehabilitation demonstrate a substantial engagement with technology and willingness to use telerehabilitation: a survey. J Physiother. 2017; 63(3):175-181.

25. Sharma U, Clarke M. Nurses' and community support workers' experience of telehealth: a longitudinal case study. BMC Health Serv Res. 2014;14(1):164.

26. Odeh B, Kayyali R, Nabhani-Gebara S, Philip N. Implementing a telehealth service: nurses' perceptions and experiences. Br J Nurs. 2014; 23(21):1133-1137.
27. Rykkje L, Hjorth GHB. "Safety at Home": experiences from testing of video communication between patients and home health care personnel. Sage Open. 2017;7(4):1-11.

28. Brewster L, Mountain G, Wessels B, Kelly C, Hawley M. Factors affecting front line staff acceptance of telehealth technologies: A mixed-method systematic review. $J$ Adv Nurs. 2014;70(1): 21-33.

29. Michie S, Johnston M, Abraham C, et al. Making psychological theory useful for implementing evidence based practice: a consensus approach. Qual Saf Health Care. 2005;14(1):26-33.

30. Dyson J, Lawton R, Jackson C, Cheater F. Does the use of a theoretical approach tell us more about hand hygiene behaviour? The barriers and levers to hand hygiene. J Infect Prev. 2011;12(1):17-24.

31. Grol R. Improving Patient Care: The Implementation of Change in Health Care. 2nd ed. West Sussex: Wiley-Blackwell; 2013.

32. Winter S, Nielsen V. Implementation of Politics. 1st ed. Copenhagen: Gyldendal; 2008.

33. Cane J, O'Connor D, Michie S. Validation of the theoretical domains framework for use in behaviour change and implementation research. Implement Sci. 2012;7(1):37.

34. World Medical Association. WMA Declaration of Helsinki - Ethical Principles for Medical Research Involving Human Subjects - WMA The World Medical Association. Available from: https://www.wma. net/policies-post/wma-declaration-of-helsinki-ethical-principles-formedical-research-involving-human-subjects/. Accessed December 13, 2017.

35. Gilbert N. Researching Social Life. London: Sage Publications; 2001:406.

36. Taylor SJ, Bogdan R. Introduction to Qualitative Research Methods: The Search for Meaning. Vol. 34. Honoken: John Wiley \& Sons, Inc.; 1985.

37. Krippendorff K. Content Analysis: An Introduction to Its Methodology. 2013:18-96.

38. Meyer S, Ward P. 'How to' Use Social Theory Within and Throughout Qualitative Research in Healthcare Contexts. Sociol Compass. 2014;8(5):525-539.

39. Brunton L, Bower P, Sanders C. The Contradictions of Telehealth User Experience in Chronic Obstructive Pulmonary Disease (COPD): A Qualitative Meta-Synthesis. PLoS One. 2015;10(10):e0139561.

40. Fairbrother P, Pinnock H, Hanley J, et al. Continuity, but at what cost? The impact of telemonitoring COPD on continuities of care: A qualitative study. Prim Care Respir J. 2012;21(3):322-328.

41. Mair FS, Hiscock J, Beaton SC. Understanding factors that inhibit or promote the utilization of telecare in chronic lung disease. Chronic Illn. 2008;4(2):110-117.

42. Hibbert D, Mair FS, May CR, et al. Health professionals' responses to the introduction of a home telehealth service. $J$ Telemed Telecare. 2004;10(4):226-230.

43. Ure J, Pinnock H, Hanley J, et al. Piloting tele-monitoring in COPD: A mixed methods exploration of issues in design and implementation. Prim Care Respir J. 2012;21(1):57-64.

44. Taylor J, Coates E, Brewster L, Mountain G, Wessels B, Hawley MS. Examining the use of telehealth in community nursing: identifying the factors affecting frontline staff acceptance and telehealth adoption $J$ Adv Nurs. 2015;71(2):326-337.

45. Sølling KI, Carøe P, Lindgren K, Mathiesen KS. Online Communication and Chronic Obstructive Pulmonary Disease (COPD). Stud Health Technol Inform. 2015;216:910.

46. Horton K. The use of telecare for people with chronic obstructive pulmonary disease: implications for management. Nursing. 2008;16(2):173-180.

47. Inskip JA, Lauscher HN, Li LC, et al. Patient and health care professional perspectives on using telehealth to deliver pulmonary rehabilitation. Chron Respir Dis. 2018;15(1):71-80.

48. Graneheim UH, Lundman B. Qualitative content analysis in nursing research: concepts, procedures and measures to achieve trustworthiness. Nurse Educ Today. 2004;24(2):105-112. 
49. Kvale S. InterViews: An introduction to qualitative research interviewing. 1st ed. Copenhagen: Sage; 1996.

50. Morgan DL. Focus groups as qualitative research. Sage Publ. 1997: $32-46$.

51. Krueger RA. Focus Groups: A Practical Guide for Applied Research. 4th ed. Sage; 1994.
52. Kidd PS, Parshall MB. Getting the focus and the group: enhancing analytical rigor in focus group research. Qual Health Res. 2000; 10(3):293-308.

53. Belzile JA, Öberg G. Where to begin? Grappling with how to use participant interaction in focus group design. Qual Res. 2012;12(4): 459-472.

\section{Publish your work in this journal}

The International Journal of COPD is an international, peer-reviewed journal of therapeutics and pharmacology focusing on concise rapid reporting of clinical studies and reviews in COPD. Special focus is given to the pathophysiological processes underlying the disease, intervention programs, patient focused education, and self management protocols.

\section{Dovepress}

This journal is indexed on PubMed Central, MedLine and CAS. The manuscript management system is completely online and includes a very quick and fair peer-review system, which is all easy to use. Visit $\mathrm{http} / / / \mathrm{www}$.dovepress.com/testimonials.php to read real quotes from published authors. 